DEMOGRAFIE

\title{
Die Unternehmen wetterfest machen
}

\author{
Christian Tölken
}

Angesicht der Bevölkerungsentwicklung muss die Sozialwirtschaft dringend demografiefeste Personalstrategien entwickeln, wenn sie ihre Leistungsversprechen gegenüber Nutzern, Finanziers, Politik und Gesellschaft auch in Zukunft einlösen will.

Kaum noch hören können wir den Satz » Die Mitarbeitenden sind unser wertvollstetes Kapital «, so oft wurde er schon beschworen. Doch nun spricht alles dafür, dass dieses Kapital aufgezehrt wird, bevor einige erkannt haben, dass es da wirklich ungenutztes Kapital gibt. Demografie bedeutet für die Sozialwirtschaft: »Wer wird morgen die Alten und Behinderten pflegen und fördern? « Demografie stellt auch die Frage, ob Teile der Sozialwirtschaft überleben können. Und all das in einem überschaubaren Zeitraum von weniger als dreißig Jahren.

Das Erwerbspersonenpotenzial in Deutschland geht bereits bis 2020 stark zurück. Ältere Arbeitnehmer werden schon bald diejenigen unter 30 Jahren dominieren. Vielfach unbeachtet, aber noch bedeutender als die quantitative ist die qualitative Veränderung des Arbeitskräfteangebots durch die schleichende Veränderung der Altersstruktur. Bereits jetzt zeichnet sich ab: Neben der deutlichen Alterung der Belegschaften wird der Nachwuchs junger, qualifizierter Arbeitskräfte weitgehend versiegen. Die Sozialwirtschaft wird sich in einem scharfen Wettbewerb um Fachkräfte und qualifizierten Berufsnachwuchs wiederfinden. Diese Entwicklungen sind unumkehrbar. Folge: Alle gegebenen Leistungsversprechen sind gefährdet.

Demografiefeste Personalstrategien können helfen. Aber natürlich umfassen personalstrategische Überlegun- gen sehr lange Zeiträume, bis sie gereift sind und Früchte tragen. Auch müssen kurzfristig angelegte Planungsgewohnheiten des Personalmanagements dabei überwunden werden. Wunder sind nicht zu erwarten, allerdings kann so Nachhaltigkeit in der Personalwirtschaft gewonnen werden. Wer gewohnt ist, KostenNutzen-Gesichtspunkte zum Maßstab aller betrieblichen Prozesse zu machen, wird nur schwerlich das Thema Demografie hier einordnen können. Das ist ein Fehler. Demografie ist ein Querschnittsthema, das man unschwer quantifizieren kann. Einzelaspekte, wie etwa Projektionen der Fluktuation oder des Krankenstandes und der sich daraus ableitenden Kosten eignen sich sehr gut, um die wirtschaftlichen Folgen zu beziffern.

Vielen ist Demografie ein diffuses Thema, weil die schwerwiegenden Folgen »ja erst morgen « eintreten werden. Alles sei im Lot, das beschwören die Verantwortlichen in der Sozialwirtschaft immer wieder. Auch dann, wenn es jetzt schon schwieriger wird, geeignete Fachkräfte $\mathrm{zu}$ finden, mit denen man die gegebenen Leistungsversprechen halten kann. Oft ist auch die vielfach festzustellende gestauchte Alterspyramide hochgradig nützlich, eignet sie sich doch als sanftes Ruhekissen. Scheinbar besteht aktuell kein Handlungsbedarf. Brechen wir jedoch die demografische Entwicklung auf konkrete betriebliche Handlungsfelder herunter, dann löst sich die Diffusität auf: Was bedeuten Demografie und Personalgewinnung, Demografie und Personalentwicklung, Demografie und Gesundheitsschutz tatsächlich?

Wen die gegenwärtige Altersstruktur beruhigt, der darf wirklich aufwachen. In zehn Jahren werden aus den jungen zwischen 20 bis 30 Jahren Arbeitskräfte mittleren Alters und aus den heute 40 bis 50-Jährigen Ältere

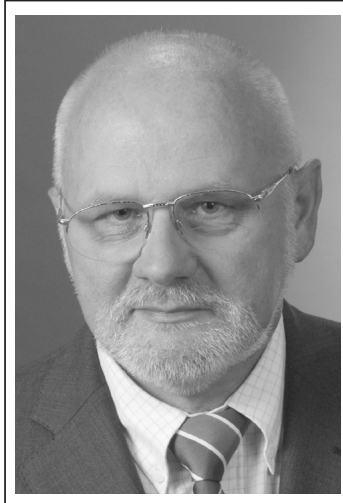

Christian

Tölken

(59) ist

Senior-

Partner

der Consolutions $\mathrm{GmbH} \&$ Co. $K G$. Die Organisation berät Un-

ternehmen und Institutionen aus dem Sozial- und Gesundheitswesen. Tölken kümmert sich vor allem um die Themen Personalwirtschaft und Personalentwicklung (LeadershipEntwicklung, Begleitung und Entwicklung von Führungskräften und Führungsnachwuchs, Mentoringprogramme, Coaching) sowie Fragen der Unternehmensführung einschließlich der Interimsgeschäftsführung. Vom Standort Berlin aus hat er außerdem Kontakte zur Bundesregierung, den Bundestagsfraktionen und der Europäischen Kommission ausbauen. Tölken war 25 Jahre Mitarbeiter der Rummelsberger Anstalten, zuletzt als Hauptgeschäftsführer.

geworden sein. Die heute über 50 sind, werden ausscheiden oder bereits ausgeschieden sein. Der eintretende Wissensverlust kann nicht aufgefangen werden, weil der Nachwuchs an jüngeren Fachkräften versiegt. Auch zeigt die Fluktuation, dass ein viel zu großer Anteil von Nachwuchskräften, bereits innerhalb der ersten vier Jahre nach Abschluss ihrer beruflichen Qualifizierung wieder ausscheidet. Wer sich dies vor Augen führt, kann personalpolitische Fehlentwicklungen und Risiken so viel klarer erkennen und Gegenmaßnahmen ergreifen. Er kann das Unternehmen wetterfest machen und steuern.

\section{Wir leben in einer Gesellschaft des langen Lebens}

... und zugleich in einer Gesellschaft, die Ältere viel zu früh aus der 
Aktivität in die Abhängigkeit entlässt. Die Deutschen haben immer noch ein falsches Menschenbild, weil sie den älter werdenden Menschen nichts zutrauen. Wie sie auch den Menschen mit einer Behinderung nichts mehr zutrauen. Beides ist falsch, es ist inhuman. Wir begehen gleich zwei Kardinalfehler. Viele Unternehmen verzichten auf die beruflichen Fähigkeiten der älteren Mitarbeitenden, weil sie ihnen einfach zu teuer sind. Und die Jüngeren müssen immer größere finanzielle Lasten schultern, ohne dass sie ausreichend verdienen.

Das ist die Lage. Das Potenzial der Erwerbsfähigen nimmt bis 2040 stark ab. In wenigen Jahren wird über die Hälfte aller Mitarbeiter älter als 50 Jahre sein. Darauf sind nur wenige Unternehmen vorbereitet. Über 40\% aller deutschen Unternehmen haben heute gar keine Mitarbeiter, die über 50 Jahre alt sind. Nur in den Aufsichtsgremien und den Vorständen sieht das anders aus. Unternehmen, die heute keine Mitarbeiter über 50 haben und nicht stark wachsen, sehen sich darüber hinaus einer »Blockalterung « gegenüber: Das Durchschnittsalter solcher Belegschaften wird jährlich um jeweils ein Jahr wachsen. Die Altersstruktur innerhalb der Belegschaften nimmt immer mehr die Form gestauchter Alterspyramiden an. Einem breiten Mittelbau stehen die schmal besetzten Beschäftigtensegmente der Jüngeren und Älteren gegenüber. Der Mittelbau umfasst heute fast die Hälfte der Beschäftigten. Dieser disproportionale Altersaufbau stellt gegenwärtig kaum ein Problem dar, weil die Mittelalten unter Innovations- und Leistungsgesichtspunkten die Aktivposten sind. Auf ihren Schultern ruht der Erfolg der Unternehmen. Denkt man jedoch die Entwicklung nur ein paar Jahre weiter und lässt den »Berg an Mittelalten « durch die Jahrgänge wandern, dann ist in absehbarer Zeit der »Altersberg « das quantitativ bedeutendste Beschäftigtensegment. (1)

\section{Wir müssen umdenken, wenn wir die Sozialwirtschaft wetterfest machen wollen}

Was überhaupt ist ein älterer Mitarbeiter? Ganz modern betrachtet: Ein älterer Mitarbeiter ist jeder, der in den letzten fünf Jahren weder die
Stelle gewechselt noch eine entwicklungsorientierte Weiterbildung absolviert hat - auch wenn er erst 38 ist. Immer noch denken wir ganz anders und falsch: Regelmäßig wird der ältere Mitarbeiter über das Lebensalter definiert, meist wird die Bezeichnung den über 50- Jährigen zuerkannt. Der Begriff hat etwas abwertendes - genau deshalb würden sich Unternehmensleitungen auch dagegen verwahren, als »ältere Mitarbeiter « bezeichnet zu werden. Was haben alle 50-Jährigen, von ihrem Alter abgesehen, gemeinsam? Objektiv nichts außer ihrem Image.

Untersuchungen zeigen uns: Ein 70Jähriger ist körperlich und geistig so fit wie ein 60-Jähriger im Jahr 1965. Dadurch wird der Anteil der Jungen und Junggebliebenen erhöht. (2) die Motivation sind die das Berufsleben bestimmenden Faktoren. Soll das alles nichts gelten, wenn es um die Beurteilung der Älteren und ihrer Chancen in den Unternehmungen geht? Ältere sind grundsätzlich nicht weniger leistungsfähig als Jüngere. Das Problem ist ja nicht die Leistungsfähigkeit an sich, sondern die Leistungsfähigkeit im Verlauf der Lebensarbeitszeit. Der eintretende Verschleiß der Arbeitskraft ist die Wirkung eines lang andauernden gesundheitlich riskanten Gebrauchs der Arbeitskraft. Die bittere Wahrheit ist doch: Viel zu viele können in den Unternehmungen auch der Sozialwirtschaft gar nicht alt werden. Weil die aktuellen betrieblichen Strukturbedingungen und auch die Einstellung Älteren gegenüber das nicht zulassen. In aller Kürze muss sich das

\section{»Ein älterer Mitarbeiter ist jeder, der sich nicht weiterentwickelt - auch wenn er erst 38 ist «}

Aber immer noch gelten Ältere als motivationsmüde, betriebsblind, unflexibel, häufig krank und wenig lernfähig. Das Gleichstellungsgesetz lässt grüßen!

Auch muss das nicht an den älter gewordenen Menschen liegen, wenn es stimmen würde. Darum und weil sie scheinbar nur noch eine kurze »Restlaufzeit « haben, werden auch in der Sozialwirtschaft über 50- Jährige bei der Besetzung wichtiger Stellen übergangen. So bestätigen sich die Vorurteile im Sinne einer sich selbst erfüllenden Prophezeiung: Ein älterer Mitarbeiter ist ein Mitarbeiter ohne berufliche Perspektiven. Damit wächst die Gefahr, dass alle anderen oben aufgezählten negativen Eigenschaften auftreten. All das ist natürlich blanker Unsinn. Objektiv lässt sich keine der aufgezählten negativen Eigenschaften für den Durchschnitt der über 50-jährigen Menschen bestätigen. Die Lernfähigkeit als solche lässt nicht nach. Die didaktischen Konzepte müssen allerdings angepasst werden.

Erfahrungshintergrund, das erworbene Wissen, die Lernerfahrung und ändern, wenn die deutsche Sozialwirtschaft nicht absaufen will.

\section{Das ist die Aufgabe: Mit demogra- fiefesten Strategien das Überle- ben sichern}

Die immer noch vorherrschende passive Verwaltung des Personals muss abgelöst werden durch ein aktives und strategisches Management der knappen Ressource Mensch. Sozialwirtschaftliche Unternehmen, die bereits heute die denkbaren Strategien nutzen, werden in wenigen Jahren einen strategischen Vorsprung haben. Es geht um Strategie. Das war schon immer die Kunst zur rechten Zeit die richtigen Dinge zu tun.

Um bestehen zu können, muss die Sozialwirtschaft alle betrieblichen Maßnahmen auf eine arbeitslebenslange Entwicklung richten. Die Grundstrategie dabei ist: Fast alle Aktivitäten zugunsten jüngerer Mitarbeiter kommen auch älteren Mitarbeitern zugute und umgekehrt. Je früher langfristige Weiterbildung und gesundheitliche Prävention einset- 
zen, umso größer sind die Erfolge. Das schließt auch familienfreundliche Maßnahmen mit ein. Familienfreundlichkeit rechnet sich, wenn es um pragmatische Lösungen, wie flexible Arbeitszeitmodelle geht oder eine Führungskultur, die Vereinbarkeit von Familien- und Berufsleben zulässt.

Auch in Zukunft geht es um die zentralen unternehmerischen Ziele der Leistungs- und Effizienzsteigerung sowie des Erhalts und Ausbaus der Innovationsfähigkeit. Die wesentlichen Personalstrategien betreffen ohnehin weniger den älteren Mitarbeiter selbst, sondern sein Ansehen im Unternehmen. Sie werden maßgeblich durch Gesundheit, Leistungsfähigkeit und Leistungsbereitschaft der Beschäftigten beeinflusst. Deswegen ist es auch überlebensnotwendig, die Fähigkeiten und Kompetenzen der Mitarbeitenden sowie das, was sie gesund erhält, wahrzunehmen, auszubauen und zu unterstützen. Dazu stehen uns unterschiedliche Strategien zur Verfügung, um Arbeit gesundheits- und alternsgerecht zu gestalten. Drei Prämissen leiten eine nachhaltig angelegte alternsgerechte betriebliche Personal- und Gesundheitspolitik:

1. Die Anforderungen an die Arbeit wandeln sich immer schneller und das bleibt nicht ohne Folgen für die Arbeitstätigen.

2. Eine aktive Gestaltung von Erwerbsverläufen fördert die Leistungsfähigkeit nachhaltig.

3. Die Beschäftigten sind die zentralen Akteure des Wandels.

Innovation und Erfahrung sind die Schlüssel für Erfolg und Zukunft der Sozialwirtschaft. Das gilt auch für älter werdende Mitarbeitende. Die Sozialwirtschaft muss alles dafür tun, um ihre Mitarbeiterinnen und Mitarbeiter erfolgreich zu halten, belastbar zu sein und engagiert Herausforderungen anzunehmen. Das bedeutet für die Beschäftigten: lebenslanges Lernen und Zukunftschancen nutzen. Für die Unternehmungen bedeutet das: ältere Beschäftigte besser einsetzen, fortbilden und unterstützen.

Die auch in der Sozialwirtschaft zunehmende Flexibilisierung und Dezentralisierung der betrieblichen $\mathrm{Ab}$ läufe führt dazu, dass die hier Tätigen eine höhere Eigenverantwortung einbringen müssen. Das können sie auch und viele wollen das auch. Sie sind bereits jetzt immer mehr selbst verantwortlich dafür, wie sie ihre Arbeit organisieren und ausführen. Damit entscheiden sie selbst auch immer stärker darüber, inwieweit gesundheitsriskant oder gesundheitsschonend gearbeitet wird. Deswegen müssen sie beteiligt werden an allen Personalstrategien.

Nicht vergessen werden dabei darf, dass die Leistungsfähigkeit ein individueller Parameter ist und somit auch einer individuellen Betrachtung bedarf. Deswegen sollten alle Strategien in der Umsetzung von einem stärkenund ressourcenorientierten Ansatz ausgehen, der es ermöglicht im Alter spezifische Stärken zu entwickeln und Leistung zu erhalten. Unser negativ besetztes Bild vom alternden Arbeitnehmer versperrt uns jedoch oft die Sicht auf diese spezifischen Stärken und Kompetenzen, die in unserer Gesellschaft sinnstiftend eingesetzt werden könnten.

Natürlich ist die Demografie als Trend nicht einfach vom Himmel gefallen. Wie die meisten relevanten Trends ist sie das Ergebnis von Veränderungsprozessen. Wir müssen sie jedoch einordnen. Ganz sicher werden wir in den nächsten Jahren einen substanziellen Perspektivwechsel vollziehen müssen. Es gibt ja noch weitere Entwicklungen, die unser Leben bestimmen immer mehr bestimmen: Die Globalisierung fordert von jedem ein flexibles Engagement und ein hohes Maß an Mobilität. Dadurch verändern sich die Beziehungen zur Mitwelt, unser Berufsalltag. Die Rollenerosion zwischen den Geschlechtern macht neue Modelle, wie Beziehungen und Intimität künftig gelebt werden können, notwendig. Die Erwerbsarbeit wird für Männer und Frauen zum selbstverständlichen Teil des eigenen Lebensentwurfs. Dadurch verändert sich die Gestaltung von Familie und Privatleben.

Klar ist in jedem Fall: In dieser Situation ist unsere Sozialwirtschaft ganz schön gefordert. Neben der Sicherung ihrer Leistungsbereitschaft wird sie Dienstleistungen zur Unterstützung entwickeln müssen, die es erlauben, der neuen Realität zwischen Alter, Familie und Beruf gerecht zu werden.

\section{Anmerkungen}

(1) Gereon Stock und Karl Heinz Kolz, Zukunftsszenarien der Personalpolitik, in: Das Personalmagazin 10/2006, S. 16-18.

(2) Schwentker, Björn, Aussterben abgesagt. Deutschland hat die Demografie entdeckt, in: Die Zeit Nr. 24, 2006, Seite 35-36.

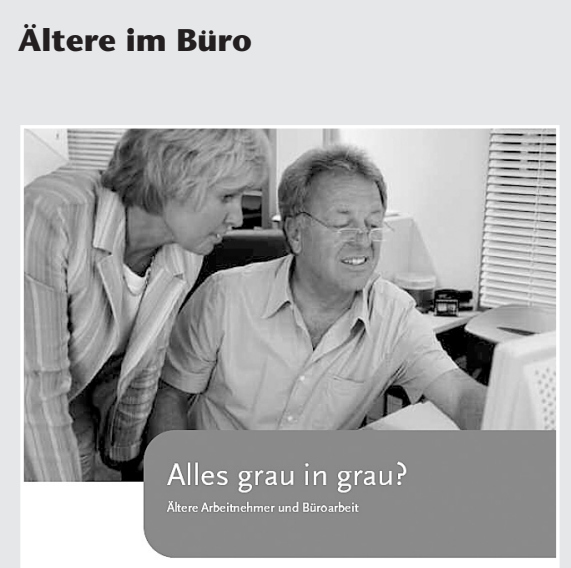

Das Altern der Belegschaften betrifft in großem Umfang die Büroarbeiter: 17 Millionen von 39 Millionen Erwerbstätigen arbeiten am Schreibtisch. Auch und gerade Unternehmen, die von Büroarbeit geprägt sind, müssen den demografischen Wandel gestalten. Über Hintergründe und Handlungsmöglichkeiten informiert eine neue Broschüre der Bundesanstalt für Arbeitsschutz und Arbeitsmedizin und der Initiative Neue Qualität der Arbeit. Die Schrift zeigt auch praktische Beispiele, wie Büroarbeit altersgerecht gestaltet werden kann. Im Mittelpunkt stehen dabei die Handlungsfelder »Sicherheit und Gesundheit «, »Arbeitsorganisation und Arbeitsgestaltung «, »Unternehmenskultur und Führungsverhalten« sowie »Qualifizierung, Weiterbildung und lebenslanges Lernen«. Die 54-seitige Publikation »Alles grau in grau? Ältere Arbeitnehmer und Büroarbeit « kann kostenlos als PDF-Datei aus dem Internet heruntergeladen werden.

Internet http://www.baua.de/nn_21604/de/Publikationen/Broschueren/A46,xv=vt.pdf 